\title{
ARTICLE
}

$\mathrm{SCl} \&$ Exercise

\section{Critical factors influencing the decision to enroll in a physical activity intervention among a predominant group of adults with spinal cord injury: a grounded theory study}

\author{
Byron W. Lai $\mathbb{D}^{1,2} \cdot$ James H. Rimmer ${ }^{2,3} \cdot{\text { Alex } \text { Yates }^{2} \cdot \text { Amanda Jeter }^{2} \cdot \text { Hui-Ju Young }}^{4} \cdot$ Mohanraj Thirumalai $^{5}$. \\ Tapan Mehta ${ }^{5} \cdot$ Jereme Wilroy ${ }^{6}{ }^{6}$
}

Received: 21 February 2020 / Revised: 17 July 2020 / Accepted: 20 July 2020 / Published online: 3 August 2020

(c) The Author(s), under exclusive licence to International Spinal Cord Society 2020

\begin{abstract}
Study design Grounded theory qualitative approach.

Objectives To examine critical factors associated with interest in enrolling in a physical activity (PA) research intervention among a predominant group of adults with spinal cord injury (SCI) and develop a theory that can enhance future recruitment success.

Setting Participants were recruited through the network of a community exercise facility for people with physical disabilities.

Methods Interviews were conducted with 22 wheelchair users (mean age $46 \pm 13$ years; SCI [ $n=19]$, cerebral palsy [ $n=1$ ]; multiple sclerosis $[n=1]$; and bilateral limb loss $[n=1])$ in either a one-on-one format or focus group. Interview data were coded, and these codes were organized into conceptual categories using a constructivist grounded theory framework.

Results Adults with SCI conceive three core concerns with enrolling into a PA trial: (1) capability to participate in the program due to scheduling, transportation, and secondary health conditions; (2) mental balancing of anticipated benefits versus the difficulty of starting the program; and (3) desirability of the program characteristics based on their preferences and needs. Concerns were organized into a theory that may enhance future recruitment success.

Conclusions Recruitment is often overlooked in PA research for people with SCI despite it being a primary rate-limiting factor that severely limits the external validity of published studies. Study findings identified core recruitment concerns that are likely similar with general barriers to PA participation. This paper proposed a 3-step decision-making process that can serve as a starting point for overcoming recruitment issues in PA research with people with SCI.
\end{abstract}

Supplementary information The online version of this article (https:// doi.org/10.1038/s41393-020-0530-7) contains supplementary material, which is available to authorized users.

Byron W. Lai

byronlai@uab.edu

1 Division of Pediatric Rehabilitation Medicine, University of Alabama at Birmingham/Lakeshore Foundation Research Collaborative, Birmingham, AL, USA

2 Lakeshore Foundation, University of Alabama at Birmingham/ Lakeshore Foundation Research Collaborative, Homewood, AL, USA

3 Dean's Office, University of Alabama at Birmingham/Lakeshore Foundation Research Collaborative, Birmingham, AL, USA

\section{Introduction}

National datasets demonstrate that people who use wheelchairs as a primary means of mobility are largely not participating in levels of physical activity (PA) that can provide health-related benefits [1-4]. Despite substantial growth in

4 Department of Physical Therapy, University of Alabama at Birmingham/Lakeshore Foundation Research Collaborative, Birmingham, AL, USA

5 Department of Health Services Administration, University of Alabama at Birmingham/Lakeshore Foundation Research Collaborative, Birmingham, AL, USA

6 Department of Physical Medicine and Rehabilitation, University of Alabama at Birmingham/Lakeshore Foundation Research Collaborative, Birmingham, AL, USA 
published clinical studies demonstrating benefits to physical fitness, function, and well-being among people with disabilities [5-9], research studies still struggle with recruiting people with SCI into PA trials [10].

Barriers to exercise participation have been long established among people with SCI and other groups who use wheelchairs. A systematic review by Martin Ginis et al. [11] identified that barriers were multifaceted and crossed all levels of the socioecological model. Barriers included intrapersonal (e.g., employment, psychological factors, and body functions and structures), interpersonal (e.g., social support, attitudes of others, and social processes), institutional (e.g., facility or organization processes, structural designs, and program factors), community (e.g, relationships among groups and organizations, products and technology for exercise, and climate), and policy (e.g., funding for programs, transportation, and professional and staff training). While these barriers certainly explain participation in community exercise, there are no published theory-based studies on how people with SCI decide to participate in PA intervention research. Given that theory-based strategies are effective in promoting PA behavior throughout an intervention [12], theories should also underpin strategies that can enhance recruitment success.

High-quality confirmatory evidence (i.e., randomized controlled trials and meta-analyses) can expedite the translation of carefully controlled clinical interventions into real-world community-settings, but PA studies have been consistently impeded by an inability to enroll large enough samples of people with SCI or wheelchair users to reach an adequate level of external validity $[5,6]$. A recent scoping review found that only $42 \%$ of adults with physical disabilities who were contacted agreed to enroll in a PA trial [10]. Common reasons for nonparticipation included unspecified refusal to participate, exclusion due to wheelchair use, difficulties with travel, unavailable transportation, and scheduling concerns [10]. However, further detail is required to create targeted strategies that increase enrollment among people with SCI and others who use wheelchairs. Thus, the purpose of this study was to explain critical factors associated with the decision-making process to enroll/not enroll in a home-based PA research study among people who use wheelchairs. Specific influencers of interest were barriers and facilitators to starting a PA program, as well preferences for the design of PA interventions.

\section{Methods}

The design was a qualitative grounded theory study that included both one-on-one and focus group interviews, based on Charmaz's constructivist grounded theory framework [13]. This method posits that theories are constructed "through our past and present involvements and interactions with people, perspectives, and research practices" [13]. Accordingly, all study components were guided by the following philosophical assumptions: a critical realism ontological perspective (i.e., there is a singular reality that can be understood through subjective perceptions or experiences of events that are linked with the reality) [14] and an interpretivism epistemological perspective (i.e., knowledge is socially constructed by both the participant and researcher) [15]. In other words, wheelchair users recalled or perceived a reality of deciding to start a research study, and our research team acknowledged that perceptions of events that were linked with this reality were recreated by the participant, as well as the interaction between the participant and the researcher.

\section{Participants}

Regarding eligibility, adults ( $>17$ years) had to use a wheelchair as a primary means of mobility and been previously contacted to participate in PA research. The research team decided (a priori) to halt recruitment if one of two criteria were reached: (1) a convenience sample of 30 people who used wheelchairs; or (2) theoretical saturation (i.e., the data analysts felt confident that conceptual categories were adequately represented and explored) [13]. For the one-on-one interviews, participants were recruited from a database of people with SCI who were unable to enroll in an onsite PA program. To reach the target sample size, the team recruited people with SCI through the network of an adapted fitness facility in Birmingham, Alabama, USA. While the target group was people with SCI, we also recruited three people with either cerebral palsy $(n=1)$, multiple sclerosis $(n=1)$, and bilateral limb loss $(n=1)$. The rationale for including these three additional participants is that they were identified as wheelchair users and were likely to experience similar factors that influence the decision to enroll or not enroll in a PA intervention [11].

\section{Procedures}

One-on-one interviews were conducted via a single telephone call (1-h max duration) by one interviewer (BL). Each interview was guided by 10 open-ended questions with subsequent follow-up questions. Questions were designed to probe factors and thought processes that likely affected a person's decision to start a PA intervention, including barriers and facilitators to starting a program, general perceptions of and preferences for PA, perceptions of their current level of PA, and options for PA within the community (see Appendix 1 for the interview guide). Prior to starting the interview, participants were asked to report 
their age, sex, ethnicity, and disability and position themselves in a quiet and comfortable setting. Participants were thanked with a $\$ 50$ electronic gift card.

Focus group interviews were conducted by two interviewers (BL \& JW). The focus group duration was limited to $2.5 \mathrm{~h}$, and the size was limited to 10 people. Focus groups were guided by 10 questions that resembled the same topics that were covered in the one-on-one interviews. The focus groups were hosted onsite at an adapted fitness facility, in a quiet and private room. Prior to starting a focus group, participants provided written informed consent and completed a demographic questionnaire. Focus group participants were thanked with a $\$ 25$ gift card. Both the focus group and phone call interviews were audio recorded and transcribed for data analysis.

\section{Analysis}

The research team combined two qualitative research methods (focus groups and one-on-one interviews) to enhance the completeness/richness of data [16]. First, four one-on-one interviews were conducted and analyzed to develop initial concepts and patterns [17]. Second, one focus group was conducted and analyzed $(n=6)$ to use "interaction data resulting from discussion among participants to increase the depth of inquiry" [16, 18]. Third, further one-on-one interviews were conducted and analyzed $(n=7)$ to enrich initial concepts and patterns with detailed accounts of thoughts, attitudes, beliefs, and knowledge [16]. Last, a final focus group $(n=5)$ was conducted and analyzed to further enrich created concepts and to identify any potentially relevant gaps in knowledge that might require further investigation.

Data analyses were conducted by 2 authors (BL and JW) and included three overall phases: (1) generation of initial codes (i.e., phrases that represent lines of text) and (2) focused codes (i.e., phrases that represent one or more initial codes), and (3) creation of conceptual categories (i.e., higher order phrases that represent one or more focused codes) [13]. First, the analysts separately generated initial codes on a line-by-line basis for each transcript by coding actions and thought processes versus themes and topics. Audio recordings were transcribed verbatim by a third-party service (Rev.com) and checked for accuracy against the original recordings by research staff (AY). The analysts referred back to the audio recordings to understand the tone and emphasis of unclear transcript text. Second, the analysts each separately recoded their initial codes into fewer higherorder focused codes. Focused codes resembled comparisons, patterns, meanings, and relationships between initial codes. Focused codes were created, edited, and compared with the data and initial codes routinely on a case-by-case basis for every transcription. Throughout coding, the analysts recorded detailed memos of rationales, patterns, and relationships amongst codes that may inform the conceptual categories [13].

Third, after each analyst matched their list of focused codes, they periodically met to merge their results into overarching conceptual codes, which were collapsed into conceptual categories. The analysts met to merge their codes after each transition to analyze the different interview methods (one-on-one and focus group interviews). Authors matched their memos to support their perspective of potential conceptual codes and categories that best represented the initial and focused codes. The analysts reached a consensus on the final conceptual categories and codes by acting as critical friends [19]: a process in which analysts thoughtfully negotiate their perspective and ask provocative questions that encourage data to be viewed through another lens, until an agreement is reached on the most suitable result. The conceptual categories and codes were arranged into a conceptual map (substantive theory) to address the study objective. A substantive theory attempts to explain a certain context and is thus not meant to be generally applicable [20, 21]. All three phases of the coding process were guided by constant comparison (routine inspection of whether categories represented codes and whether codes represented higher order codes or categories) of the generated codes or conceptual categories with the representative data [13].

The two analysts had several years of experience with exercise research and training for people with disabilities. One analyst (BL) had a background in adapted PA and training in qualitative inquiry. The second analyst (JW) had a background in PA behavior change for people with disabilities, training in mixed-methods research, and had a spinal cord injury (SCI). Regarding backgrounds for other coauthors: JHR was an international leader in adapted PA and was the PI of several large randomized controlled trials of PA for people with disabilities; AY and AJ were project recruiters and managers for PA projects led by JHR; HY had a background in adapted PA; MT had a background in exercise technology for people with disabilities; and TM had a background in statistics and managed the recruitment databases for projects led by JHR. The authors represented a range of relevant disciplines and had recruitment experience on different PA projects for people with disabilities, which allowed them to provide useful feedback on the findings of the paper. When providing edits and feedback, all authors agreed to adopt the aforementioned philosophical assumptions for this project.

Several strategies were used to enhance the quality of this work [22]. The analysts kept an audit trail that detailed every key step of theory construction and provide a rationale for choices to develop codes. The analysts acted as critical friends. Moreover, the present study adhered to all 
eight core elements of grounded theory methodology: iteration; theoretical sampling (i.e., data is collected to help refine concepts); theoretical sensitivity (i.e., researchers approached the data with an open mind) [13]; memoing; constant comparison; theoretical saturation; the resultant theory was relevant and is modifiable; and substantive theory [23].

\section{Results}

Twenty-two wheelchair users were interviewed from oneon-one interviews $(n=11)$ and two focus groups $(n=11$; mean age $46 \pm 13$ years; 18 males $/ 3$ females/ 1 preferred not to answer; SCI $[n=19]$, cerebral palsy $[n=1]$; multiple sclerosis $[n=1]$; and bilateral limb loss $[n=1] ; 13$ Caucasian, 7 African American, 1 Hispanic, 1 American Indian). Twenty participants lived in Alabama and 2 in Louisiana. Seven participants (32\%) had an ongoing membership to a community fitness facility. Five participants completed high school; 5 completed some college without a degree; 10 completed a bachelor's or associate college degree; one completed a graduate degree. Ten participants $(45 \%)$ were employed full- or part-time.

\section{Results organization}

The results are organized into two sections. The first section describes conceptual categories and codes that represent concerns that influence the decision to join a PA program (see Appendix 2 for a complete list of initial and focused codes). The second section incorporates the resultant conceptual categories and codes into a theory, presented in a hierarchal model, that is likely to promote success in recruitment. The analysts believed the conceptual categories to be adequately saturated [13] and decided to halt recruitment for the interviews at 22 participants.

\section{Concerns influencing participation}

When deciding whether or not to join a PA research intervention, $\mathrm{SCI} /$ wheelchair users consciously or subconsciously perceived three conceptual categories that entered the decision-making process, namely, Capability, Balancing, and Desirability.

\section{Capability}

Participants noted that there were a variety of concerns that would directly affect their decision to participate in a PA intervention. We classified these under the category, capability. Conceptual codes (concerns) included scheduling, finance, and secondary conditions.
Participants quickly determined whether they could schedule time for PA among their other priorities. Time meant the time required for performing PA due to difficulties with mobility and the time required for transportation to a fitness or research facility. Participants identified several transportation burdens. Participants who had the ability to drive reported that transportation time to and from the fitness facility was a substantial hinderance. This included the time to change into fitness clothes, drive to a facility, transfer in and out of the car, find safe and accessible parking, wheel to the facility from the parking lot and back, and drive home. Other participants relied on friends or family to drive, while a few participants had no convenient means of allocating transportation. The time required for transportation was critical, since participants were often employed and/or had family care responsibilities. To ease transportation and scheduling concerns, participants suggested that PA programs should be delivered in their home and be able to be scheduled around participants' daily activities and commitments.

The second capability concern was finance. This was closely linked with scheduling. Participants determined whether their financial capacity could support PA participation. Some worked and could not afford consistent transportation to a fitness facility or any costs that would be required of a PA program. Others stated they would have to request time off work. These concerns could be eased by providing financial incentives.

Participants often experienced a number of secondary health conditions that prevented them from participating in PA. They reported physical conditions such as spasms, pressure ulcers, and infections or illnesses that impacted participation (e.g., exclusion from the study or inability to perform the required exercises or movements). In addition, they reported psychological conditions including depression, fatigue, and anxiety that lessened their desire to participate. Participants identified a need for PA programs that could safely work around and/or help them alleviate or manage secondary conditions (e.g., an assisted standing program for people with pressure ulcers).

\section{Balancing}

Participants underwent a cognitive process of balancing advantages and disadvantages that influenced their motivation to participate. Balancing was influenced by mental weighting of perceived benefits and the perceived energy required to start a PA program. Participants were interested in joining a PA program that could benefit them or other wheelchair users. Benefits referred to those that could be experienced directly from PA participation (physiologic or psychological improvements) or indirectly by furthering PA research knowledge. As stated by one focus group 
participant: "I can choose not to participate, but I'd rather be a part of the process that's helping the lives of people in this room". Participants further reported that PA could enhance their functional independence and help them live a longer, healthier lifestyle, and these were the two most highly desired benefits. PA was reported to increase strength, energy level, mood, health, and wellbeing. These benefits could lead to observable changes in secondary conditions or the performance of daily activities, which were the most highly desired benefits.

Participants stated that anticipated benefits from participating in a study should be clearly conveyed by the recruiter.

Energy level was a substantial barrier on participant motivation to engage in PA. Energy meant perceived energy expenditure, and this was acknowledged as a difficult barrier to surpass when starting a PA program. Participants recalled that the performance of daily activities with a wheelchair demanded much of their energy capacity, which was worsened by secondary health conditions. Motivation could be increased intrinsically by a belief of meaningful perceived benefits from participation and a desire for programs that incorporated social support, as well as increased extrinsically by monetary incentives.

\section{Desirability}

Participants assessed the desirability of the program based on their preferences and physical or psychological needs. Conceptual codes included personalized programs, mutual understanding, and social support. Social support was a highly desired program characteristic and was described as establishing relationships and social bonds with others and receiving physical assistance for people with greater functional impairments. Participants desired positive encouragement, mentorship, and a sense of companionship or being part of a collective, which stemmed from feelings of isolation and loneliness. They identified a lack of nearby community support groups or events and, if these situations could be identified, transportation was often unavailable.

Based upon past negative experiences, participants were highly skeptical that a research PA intervention would be suitable or personalized to their needs. The participants themselves were largely unaware of enjoyable movements or exercises they could perform safely and comfortably within a wheelchair and, instead, recalled exercises that they enjoyed prior to their injury. Participants recalled limited options and nearby places within their community that were suitable for PA. Within community facilities, they identified few accessible or usable exercise machines or equipment and often felt uncomfortable and anxious in these environments. As stated by participant 5 :
"I think one thing that just kind of scares me away is that I bet there's nothing there that I could use. But on the other hand, I haven't checked. You know, before we moved to this area, I did use the gym periodically and there wasn't a lot I could do there".

Because of these issues (and capability concerns), the home was identified as a convenient location for performing PA.

A key concept of desirability included a desire for personalized programs. As stated by participant 17, "people with disabilities are as diverse as people without disabilities", which will require just as diverse strategies to promote PA, not the rigidly structured plans or programs that were presented to them in research-based programs (e.g., one PA program procedure versus a nonexercise control).

"I think if the participants had more opportunity and options to choose what their outcomes could be within the study, then it would create more choice and more option, which I think is something always needed. Because when you have a disability, you have one choice usually, and choice is important" (Participant 17).

Interestingly, the term "research" conjured negative perceptions. Participants sometimes perceived that researchers were an uncomfortable higher authority who treated wheelchair users as subjects, as opposed to taking the time necessary to understand and accommodate their challenges (e.g., complications and difficulties from secondary conditions). Accordingly, participants desired health professionals or promoters of PA to demonstrate credibility and trustworthiness: to communicate as a person who is part of an interdependent relationship (versus a higher authority). Credibility and trustworthiness were essential, because participants were often skeptical of what data would be collected and how the research results would help them or others. In line with these characteristics, participants reported that they would be more receptive joining a PA program if approached or contacted by a person who used a wheelchair and understood their daily challenges. To further alleviate these concerns of unease, participants recommended that researchers could familiarize wheelchair users with the value of research through community events and build partnerships with existing organizations in the community.

\section{Organizing concerns into a process that can enhance recruitment}

The research team organized participant concerns into a 3step process that that can likely promote success in recruitment. The three categories (Capability, Balancing, and Desirability) are positioned within a pyramid that resembles their importance in the decision-making process, which recruiters should target in order from bottom-to-top 
(Fig. 1). This theory was informed by seven preceding drafts of conceptual maps (see Appendix 3 for preliminary conceptual maps) and was framed upon (i.e., "grounded" on) focused codes and initial codes (Appendix 2). Based upon this theory, a bullet list of recommendations to enhance recruitment success are provided in Table 1.

Three noteworthy concepts will help in understanding the theory. First, the three categories or levels are distinct yet overlapping. For example, successful recruitment will likely depend on addressing participant concerns in order from bottom-to-top, but the lower levels act as a foundation to proceed upward and can retake precedence at any point in time [24]. Second, it is important to clarify that this model ranked concerns in the best order for promoters of PA to

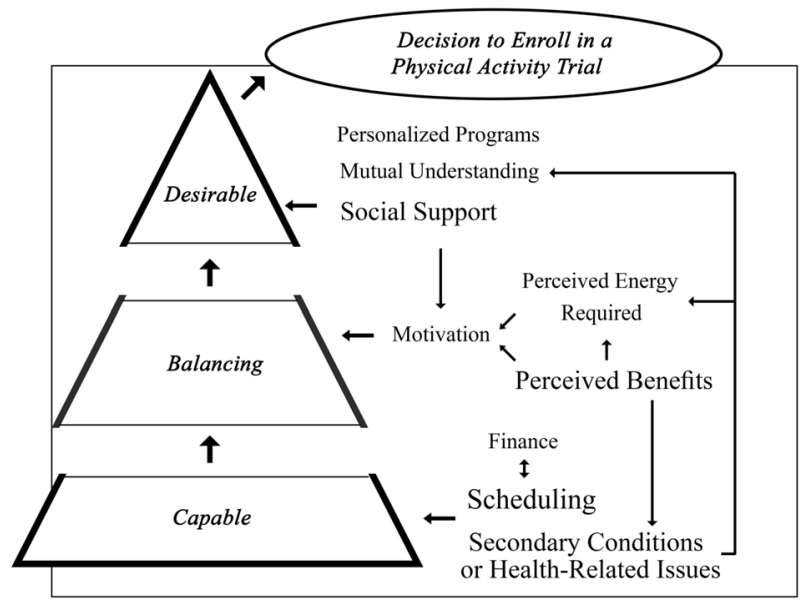

Fig. 1 3-Step decision making model for recruitment. A substantive theory explaining critical influences underlying the decision to enroll in a physical activity program. take a targeted linear approach toward recruitment, even though participants' concerns may not necessarily be linear. One level may not be more important for each individual in every situation. Last, participants were interviewed prior to the Covid-19 global pandemic, but this paper was reviewed and revised thereafter. The research team decided to retrospectively modify the hierarchical model to address potential health or safety concerns, such as Covid-19. Accordingly, the categorical code Secondary Conditions, was changed to Secondary Conditions or Health-Related Issues.

\section{Recommendations for recruitment strategies}

\section{Capability}

Capability is displayed at the bottom of the pyramid to symbolize that it is a foundation that must be addressed before other concerns. During the early stages of recruitment contact (e.g., phone call, email, flyer), people with $\mathrm{SCI} /$ wheelchair users must be quickly informed that the program can fit conveniently within their schedule and that transportation and finance burdens are addressed. Recruiters of onsite studies should provide monetary incentives, supports, or protocol modifications to overcome transportation to an onsite facility. The final capability concern, secondary health conditions or health-related, can be addressed by providing evidence-based programs that are suitable, safe and supported by knowledgeable staff who understand secondary condition symptoms and worsening factors. In the case of Covid-19 or other health-related conditions that compromise the safety of in-person data collection and

Table 1 Recommendation for recruitment strategies.

Concern Category Recommendation

Capability - Convey that the program can fit within their schedule and is not a substantial time burden

- Inform participants that measures are in place to accommodate or address transportation and financial concerns (no cost to them and financial incentives)

- Participants prefer home-based programs which could be implemented via telehealth

- If a program is conducted onsite, ensure that incentive outweigh transportation concerns

- Ensure that the program is safe for them

Balancing - If possible (allowable by IRB), inform participant of potential health benefits from participation

- State how the knowledge obtained from the study will help other people with the same disability

- Emphasize that participants will receive social support and specify how (if applicable)

Desirability - Further elaborate on social support details or meaningful relationships that will be developed through the program (if applicable)

- Explain who the program is designed for, ideally the program will be customized or even have adaptable options to suit the potential participant's needs

- The recruiter should be friendly, patient, professional, and understanding of the potential participant's situation and needs

- Mention organizations, gatekeepers, or coordinators that may provide the potential participant with a sense of trust

- If possible, the recruiter should be an individual with the same disability or conditions as the potential participant

No table legend necessary for Table 1 . 
enrollment, researchers could utilize entirely remote interventions and data collection procedures through telehealth [25]. Evidence-based PA programs (both onsite and remote) can be found in several published reviews [5, 6, 26, 27]. Of note, participants will be consider how the study aligns with their secondary conditions throughout the recruitment process, as these concerns were linked with those in the other two overarching conceptual categories (Balancing and Desirability).

\section{Balancing}

After resolving concerns regarding capability, SCI/wheelchair users should be provided with knowledge that can help them balance advantages and disadvantages that influence their motivation to participate. Motivation means "willpower", which is driven by mental weighting of perceived benefits and the energy that is required to start a PA program. A clear understanding of meaningful anticipated benefits can outweigh capability concerns and the energy required to start a PA program. Of note, study findings demonstrated that wheelchair users already valued PA benefits. These findings coincide with those reported previously in a review of barriers and facilitators among people with SCI [28]. Thus, a concise message conveying meaningful benefits to the person, others, or knowledge gained from joining the PA program may be adequate to enhance the likelihood of enrollment.

\section{Desirability}

After addressing capability and balance concerns, recruiters must convey the characteristics of the program so that potential participants can assess the desirability of joining it. Three core questions frame desirability: (1) Will I (the potential participant) be supported by others and will I build relationships with them?; (2) Do I have a mutual understanding with the recruiter of the program and is the recruiter aware of my capability and motivations?; (3) Does the program have movements or exercises that are suitable to my functional ability and will I enjoy them? Desirability is perhaps the most difficult categorical concern to address, but the most important, because a highly desirable program can outweigh other concerns. Resultant codes for desirability appeared similar with a meta-synthesis of qualitative research evidence examining participant experiences and perceptions of PA-enhancing interventions among people with mobility limitations [29].

$\mathrm{SCI} /$ wheelchair users must be informed that the program is customized to their needs (functional ability and preferences). A recruiter should explicitly mention what functional ability the program is inclusive of, instead of asking the person to describe their functional ability (i.e., allowing the wheelchair user to decide if the program is suitable). SCI/wheelchair users reported that they would like to refrain from providing as much personal data as possible during the first point-of-contact.

In addition, $\mathrm{SCI} /$ wheelchair users must perceive that the recruitment agent (e.g., phone call, flyer, email) is coming from a friendly, relatable, and trustworthy source. An immediate solution to this issue is to tailor recruitment communications and strategies to convey the desirable traits. Participants acknowledged that one solution could be to employ wheelchair users as recruiters, since they best understand the daily challenges associated with living with a disability. A long-term and more viable solution is to build partnerships with community organizations, coordinators, or gatekeepers, who have the desired traits and can understand the resources needed by people in the community. In this way, trust is gradually earned and can lead to enhanced participation in research and further partnership opportunities.

As stated by participant 17, wheelchair users will prefer to be contacted about diverse options to engage in PA, as opposed to rigid structured interventions that are typically offered by research studies. One solution is to design a study to include an adaptive intervention [30]. Adaptive interventions modify the type and dose of intervention provided based upon participant characteristics or preferences. Specifically, adaptive interventions include pre-planned changes that are based on a decision point (time point of altering the intervention plan), tailoring variables (outcomes that dictate intervention changes), and intervention options (variations in PA intervention responses, such as $\mathrm{PA}$ type, frequency, intensity, time, or delivery location) [30]. Another practical solution would be to provide wheelchair users with multiple PA interventions that they could potentially sign-up for during the recruitment call (if possible), as opposed to recruiting for a single study.

\section{Discussion}

Recruitment of SCI/wheelchair users in PA trials is often an afterthought when a researcher receives funding to conduct the study. This is a significant misstep given the challenges associated with recruiting a small population group in any one community. The present study identified critical recruitment concerns among a predominant group of people with SCI. These concerns led to the development of a theory that may enhance recruitment strategies for PA programs. Recruitment is the current rate-limiting factor for implementing high-quality randomized controlled trials in this population [5, 10] and severely attentuates external validity. 
Study findings complement previous data from a national study $(n=802)$ that surveyed enrollment facilitators and barriers to clinical trials among people with SCI [31]. Facilitators included improvement in function, opportunities to help others, and physical independence, and barriers included concerns about finance, functional decline, and possible side effects (or in the context of exercise: worsened secondary health conditions), and these factors were similar with those reported in the present study. Thus, study findings build upon knowledge of enrollment factors by ordering and arranging these factors within a process that can inform the development of targeted recruitment strategies for PA programs.

An important takeaway message from this study is that wheelchair users' decisions to join a PA study are underpinned by complex concerns that appeared similar with barriers and preferences of general participation in PA $[11,28]$. These concerns should be addressed early on in the design of a study. Upon commencing recruitment for a PA study, many study procedures such as budget allocation, data collection, and the design of the intervention are already "set in stone". These study design components will heavily influence recruitment success. Therefore, the findings from the present study should not only apply toward direct recruitment strategies, but also toward early study design (particularly desirability concerns). Potential a priori considerations can include: (1) allocating funds in grant applications or project budget proposals for participant incentives or effective recruitment strategies including hiring people with SCI as recruiters; (2) modifying PA interventions to be conducted entirely remotely at home through telehealth; and (3) designing PA programs that are adaptable to various functional abilities, enjoyable, and involve group-based or trainer-supervised activities, ideally with support from other wheelchair users.

\section{Limitations}

Given the qualitative nature of this study, the findings are not meant to be generalizable in a statistical sense toward the general population of SCI/wheelchair users. Instead, the developed theory may have analytical/theoretical generalizability (i.e., the theory itself can be of value and be of significance to other researchers) or transferability to similar settings [32]: geographic areas where people with physical disabilities have limited access to health or fitness care specialists or facilities and experience difficulties with public and personal transportation. Nevertheless, the sample may have been limited by the low representation of females and the adapted fitness facility network where they were recruited from. In addition, there were three participants who did not have a SCI. Although the research team did not observe any unique issues reported by these three participants during the interviews or transcriptions, the study findings may not adequately resemble the challenges experienced with starting a PA intervention among these underrepresented groups. A noteworthy but unavoidable limitation was that participants were asked to recall or perceive a hypothetical experience or situation, which is less reliable than other methods of examining how people think or feel such as experience sampling [33].

\section{Data availability}

The resultant codes and conceptual maps that led to the development of the substantive theory can be accessed in the Appendix.

Acknowledgements We would like to thank all the people who participated in the study.

Funding Funded by the Craig H. Neilsen Foundation, Pilot (Grant\#435556), National Institute on Disability, Independent Living, and Rehabilitation Research (NIDILRR; award\#: 90DPGE0005-0100), and Lakeshore Foundation.

Author contributions BL, JR, HY, MT, TM, and JW contributed to the study design. AY and AJ were heavily involved with recruitment, data processing (transcription), data collection, and the organization/ management of the focus group interviews. All authors contributed to the development of this paper. BW conducted the phone call interviews. JW and BL were the interviewers for the focus groups.

\section{Compliance with ethical standards}

Conflict of interest The authors declare that they have no conflict of interest.

Ethics We certify that all applicable institutional and governmental regulations concerning the ethical use of human volunteers/animals were followed during the course of this research. The procedures for this study were approved by the University Institutional Review Board and consent was obtained from participants prior to participation.

Publisher's note Springer Nature remains neutral with regard to jurisdictional claims in published maps and institutional affiliations.

\section{References}

1. Martin Ginis KA, Latimer AE, Arbour-Nicitopoulos KP, Buchholz AC, Bray SR, Craven BC, et al. Leisure time physical activity in a population-based sample of people with spinal cord injury part I: demographic and injury-related correlates. Arch Phys Med Rehabil. 2010;91:722-8.

2. van den Berg-Emons RJ, Bussmann JB, Stam HJ. Accelerometrybased activity spectrum in persons with chronic physical conditions. Arch Phys Med Rehabil. 2010;91:1856-61.

3. Carroll DD, Courtney-Long EA, Stevens AC, Sloan ML, Lullo C, Visser SN, et al. Vital signs: disability and physical activityUnited States, 2009-2012. MMWR Morb Mortal Wkly Rep. 2014;63:407-13.

4. Rocchi M, Routhier F, Latimer-Cheung AE, Martin Ginis KA, Noreau L, Sweet SN. Are adults with spinal cord injury meeting the 
spinal cord injury-specific physical activity guidelines? A look at a sample from a Canadian province. Spinal Cord. 2017;55:454-9.

5. Lai B, Young HJ, Bickel CS, Motl RW, Rimmer JH. Current trends in exercise intervention research, technology, and behavioral change strategies for people with disabilities: a scoping review. Am J Phys Med Rehabil. 2017;96:748-61.

6. Rimmer JH, Chen M-D, McCubbin JA, Drum C, Peterson J. Exercise intervention research on persons with disabilities: what we know and where we need to go. Am J Phys Med Rehabil. 2010;89:249-63.

7. Nooijen CF, Stam HJ, Sluis T, Valent L, Twisk J, van den BergEmons RJ. A behavioral intervention promoting physical activity in people with subacute spinal cord injury: secondary effects on health, social participation and quality of life. Clin Rehabil. 2017;31:772-80.

8. Martin Ginis KA, Jorgensen S, Stapleton J. Exercise and sport for persons with spinal cord injury. PM R 2012;4:894-900.

9. Hicks AL, Martin KA, Ditor DS, Latimer AE, Craven C, Bugaresti J, et al. Long-term exercise training in persons with spinal cord injury: effects on strength, arm ergometry performance and psychological well-being. Spinal Cord. 2003;41:34-43.

10. Lai B, Cederberg K, Vanderbom KA, Bickel CS, Rimmer JH, Motl RW. Characteristics of adults with neurologic disability recruited for exercise trials: a secondary analysis. Adapt Phys Act Q. 2018;35:476-97.

11. Martin Ginis KA, Ma JK, Latimer-Cheung AE, Rimmer JH. A systematic review of review articles addressing factors related to physical activity participation among children and adults with physical disabilities. Health Psychol Rev. 2016;10:478-94.

12. Ma JK, Ginis KA. A meta-analysis of physical activity interventions in people with physical disabilities: content, characteristics, and effects on behaviour. Psychol Sport Exerc. 2018;37: 262-73.

13. Charmaz K. Constructing grounded theory. 2nd ed. Thousand Oaks, California: SAGE; 2014.

14. Fletcher AJ. Applying critical realism in qualitative research: methodology meets method. Int J Soc. 2017;20:181-94.

15. Smith B, Sparks AC. Introduction: An invitation to qualitative research. In Smith B \& Sparkes AC (Eds.), Routledge handbook of qualitative research methods in sport and exercise 2016 (pp. 1-7). London, UK: Routledge.

16. Lambert SD, Loiselle CG. Combining individual interviews and focus groups to enhance data richness. J Adv Nurs. 2008;62:228-37.

17. Morgan DL. Focus groups as qualitative research. 2nd Edition. Thousand Oaks, CA: Sage Publications; 1996.

18. Duggleby W. What about focus group interaction data? Qual Health Res. 2005;15:832-40.

19. Burke, S. Rethinking 'validity' and 'trustworthiness' in qualitative inquiry: how might we judge the quality of qualitative research in sport and exercise sciences? In Routledge handbook of qualitative research in sport and exercise, 2016 (pp. 330-339). Routledge. https://doi.org/10.4324/9781315762012.ch25

20. Birks M, Mills J. Grounded theory: a practical guide. 2nd ed. Thousand Oaks, California: SAGE; 2015.

21. Bryant A, Charmaz K. The SAGE handbook of grounded theory. Thousand Oaks, California: Sage; 2007.

22. Smith B, McGannon KR. Developing rigor in qualitative research: problems and opportunities within sport and exercise psychology. Int Rev Sport Exer Psychol. 2018;11:101-21.

23. Weed M. Research quality considerations for grounded theory research in sport \& exercise psychology. Psychol Sport Exerc. 2009;10:502-10.

24. Deckers L. Motivation: biological, psychological, and environmental. 5th ed. New York: Routledge; 2018.

25. Lai B, Chiu C, Pounds E, Tracy T, Mehta T, Young H, et al. Covid-19 modifications to the TEAMS study: a remotely delivered teleassessment/teletraining protocol for a large randomized controlled trial comparing the effectiveness of a complementary and alternative medicine intervention for people with multiple sclerosis. JMIR Preprints. 04/05/2020:18415. https://doi.org/10.2196/ preprints. 18415

26. Castro O, Ng K, Novoradovskaya E, Bosselut G, Hassandra M. A scoping review on interventions to promote physical activity among adults with disabilities. Disabil Health J. 2018;11:174-83.

27. O'Brien TD, Noyes J, Spencer LH, Kubis HP, Hastings RP, Whitaker R. Systematic review of physical activity and exercise interventions to improve health, fitness and well-being of children and young people who use wheelchairs. BMJ Open Sport Exerc Med. 2016;2:e000109.

28. Williams TL, Smith B, Papathomas A. The barriers, benefits and facilitators of leisure time physical activity among people with spinal cord injury: a meta-synthesis of qualitative findings. Health Psychol Rev. 2014;8:404-25.

29. Williams TL, Ma JK, Martin Ginis KA. Participant experiences and perceptions of physical activity-enhancing interventions for people with physical impairments and mobility limitations: a meta-synthesis of qualitative research evidence. Health Psychol Rev. 2017;11:179-96.

30. Collins LM, Murphy SA, Bierman KL. A conceptual framework for adaptive preventive interventions. Prev Sci. 2004;5:185-96.

31. Anderson KD, Cowan RE, Horsewell J. Facilitators and barriers to spinal cord injury clinical trial participation: multi-national perspective of people living with spinal cord injury. J Neurotrauma. 2016;33:493-9.

32. Smith B. Generalizability in qualitative research: misunderstandings, opportunities and recommendations for the sport and exercise sciences. Qual Res Sport Exerc Health. 2018;10:137-49.

33. Csikszentmihalyi M, Larson R. Validity and reliability of the experience-sampling method. In Flow and the foundations of positive psychology, 2014 (pp. 35-54). Springer, Dordrecht. 